\title{
ON SINGULAR TRANSFORMATIONS IN REAL PROJECTIVE GROUPS.
}

BY PROFESSOR H. B. NEWSON.

(Read before the American Mathematical Society, April 28, 1900.)

A sINGULAR transformation in a continuous group has been defined as one that can not be generated from an infinitesimal transformation of the group. There have recently appeared in this country several papers* dealing with the question of singular transformations in continuous groups, and in particular in the subgroups of the projective group. The authors of all these papers use the same method, viz., Lie's theory of continuous groups, and consider the variables and parameters to be complex numbers.

In this paper it is proposed to treat those transformations in real projective groups which can not be generated from the real infinitesimal transformations of these groups. I shall make use of a radically new method of treatment, in fact an independent theory of these groups. In what follows the discussion will be limited to real projective transformations in one and two dimensions. The method admits of ready application to three and higher dimensions.

\section{$\S 1$. Real Transformations in one Dimension.}

Every projective transformation in one dimension

$$
T: \quad x_{1}=\frac{a x+b}{c x+d}
$$

can be reduced to one or other of the normal forms

$$
\text { (a) } \frac{x_{1}-A^{\prime}}{x_{1}-A}=k \frac{x-A^{\prime}}{x-A}, \quad \text { (b) } \frac{1}{x_{1}-A}=\frac{1}{x-A}+\alpha \text {. }
$$

In the first form $k$ is the cross ratio of the two invariant points $A, A^{\prime}$ and the pair of corresponding points $x, x_{1}$. For any transformation of this form the cross ratio $k$ is the same for all pairs of corresponding points. The invariant points $A, A^{\prime}$ and the cross ratio $k$ are the geometric constants of the transformation.

\footnotetext{
* Rettger, Amer. Jour. of Math., vol. 22, p. 60 ; Proc. Amer. Acad., vol. 33, p. 493; Williams, Proc. Amer. Acad., vol. 35, p. 97; Taber, Bulletin, vol. 6, p. 199, and several other papers by the same author. For references to these, see footnote on page 61 of Rettger's paper in Amer. Jour. of Math., vol. 22.
} 
There are two cases under ( $a$ ) to be considered: (1) When the points $A, A^{\prime}$ are both real, $k$ is also real; this is the hyperbolic case. (2) When the points $A, A^{\prime}$ are conjugate imaginary, $k$ is a complex number of the form $e^{i \theta}, i . e .,|k|=1$. These cases must be discussed separately. In the parabolic case $(b)$ we have a single real invariant point $A$ and a real constant $\alpha$. Every real projective transformation of one dimension is either hyperbolic, elliptic, or parabolic.

In the hyperbolic case all transformations which have the same real invariant points $A, A^{\prime}$ but different values of $k$ form a one parameter group, and $k$ is the variable parameter of the group. Two transformations $T$ and $T_{1}$ whose cross ratios are respectively $k$ and $k_{1}$, combine to form $T_{2}$ whose cross ratio $k_{2}$ is equal to the product of $k$ and $k_{1}$; thus $k_{2}=$ $k k_{1}$. The identical transformation in the group $h G\left(A, A^{\prime}\right)$ is given by $k=1$; and two distinct infinitesimal transformations in the group are given by $k=1 \pm \delta$. Every transformation of the group for which $k$ is positive and $k>1$ can be generated by repeating the infinitesimal transformation $k=1+\delta$ a sufficient number of times; every transformation for which $k$ is positive and $k<1$ can be generated from the other infinitesimal transformation $1-\delta$; the transformations of the group for which $k$ is negative are all singular transformations in the sense that they can not be generated from either infinitesimal transformation of the group.* All transformations of (1) for which the determinant

$$
\left|\begin{array}{ll}
a & b \\
c & d
\end{array}\right|<0
$$

are hyperbolic with negative $k$, and are therefore singular transformations.

In the one parameter elliptic group $e G_{1}$ two transformations also combine by multiplying their constants; thus $k_{2}=k k_{1}$, or $e^{i \theta_{2}}=e^{i \theta}$. $e^{i \theta_{1}}$, whence $\theta_{2}=\theta+\theta_{1}$. The identical transformation is given by $e^{2} \theta=1$, $i$. e., by $\theta=0$. Two infinitesimal transformations are given by $k=e^{ \pm i \delta}$. Evidently all transformations in the elliptic group $e G_{1}$ can be generated from either infinitesimal transformation of the group.

In the parabolic case we have a single invariant point $A$ and a constant $\alpha$, both of which are real. Two transformations of a one parameter group combine by adding the constants,

\footnotetext{
* The pair of inverse transformations for which $k=0$ and $\infty$ respectively need not be excluded from the group, but their exceptional character as limiting cases should be noted.
} 
thus $\alpha_{2}=\alpha+\alpha_{1}$. The identical transformation is given by $\alpha=0$, and two infinitesimal transformations have as constants $\pm \delta \alpha$. Every transformation with positive $\alpha$ in the group $p G_{1}$ can be generated from the infinitesimal transformations $+\delta \alpha$, and every transformation with negative $\alpha$ can be generated in like manner from - $\delta \alpha$. Hence there are no singular transformations in the real parabolic group $p G_{1}$.

THeOREM 1. In one dimension all real hyperbolic transformations with positive $k$, all real elliptic and parabolic transformations are non-singular; all hyperbolic transformations with negative $k$ are singular transformations.

The one parameter hyperbolic group is composed of three subdivisions as follows: all transformations with $1<k<\infty$ form subdivision I.; all with $0<k<1$ form subdivision II. ; all with negative $k$ form subdivision III. A one parameter parabolic group is composed of two subdivisions ; all transformations with positive $\alpha$ form subdivision I., and all with negative $\alpha$ form subdivision II. The one parameter elliptic group shows no such subdivisions.

One of the foundation stones of Lie's theory of continuous groups is that every one parameter continuous group contains one infinitesimal transformation, and that all finite transformations of the group can be generated from the infinitesimal transformation of the group. We have seen that the real one parameter groups $h G_{1}, e G_{1}$, and $p G_{1}$ each contain two distinct infinitesimal transformations, one positive and the other negative. There is no vital contradiction here, for in Lie's theory no distinction is made between positive and negative infinitesimal values of the variable parameter of a group : in the most general form of his theory the parameter is a complex variable, so that positive and negative distinctions are out of place. However, for real groups the distinction between the two infinitesimal transformations of a one parameter group requires recognition.

We have seen that all elliptic and parabolic one parameter groups can be generated from infinitesimal transformations belonging to those groups, but all the transformations of a hyperbolic group can not be so generated. Yet the hyperbolic one parameter group $h G_{1}$ is clearly continuous. It follows at once without further elaboration that the continuity of a real one parameter projective group is not coextensive with its generation from an infinitesimal transformation of the group. 


\section{\$2. Real Projective Transformations in the Plane.}

We come now to the consideration of real projective transformations or collineations in the plane. Every projective transformation of the plane belongs to one of the five types enumerated by Lie in his "Continuierliche Gruppen," page 66. The normal forms of the equations of these five types were given by the writer in the Kansas University Quarterly, Series A, vol. 7, pages 43-66. Making use of these normal forms we can determine the structure and properties of the continuous groups of projective transformations in the plane in a manner similar to that used above for one dimension.

Type I. What I have called the implicit normal form for a transformation $T$ of type $I$ is given by the equations

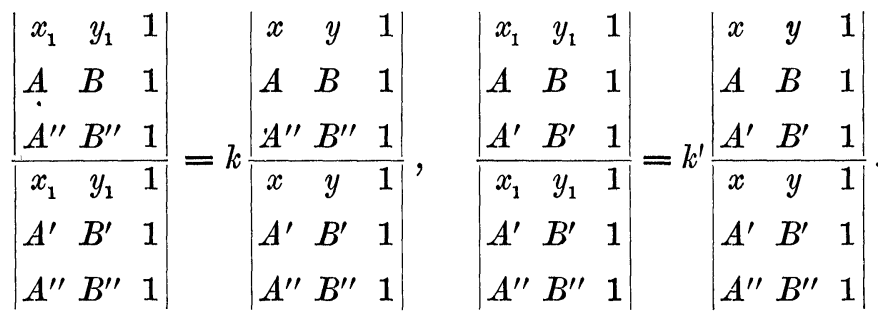

Here $A, B ; A^{\prime}, B^{\prime}$; and $A^{\prime \prime}, B^{\prime \prime}$ are the coördinates of the three vertices of the invariant triangle of $T$, and $k$ and $k^{\prime}$ are two independent cross ratios ; $k$ is the cross ratio of the one dimensional transformation along the invariant line joining $(A, B)$ and $\left(A^{\prime}, B^{\prime}\right) ; k^{\prime}$ is the cross ratio of the one dimensional transformation along the line joining $(A, B)$ and $\left(A^{\prime \prime}, B^{\prime \prime}\right)$.

There are two cases to be considered. The invariant triangle is either real in all its parts or has one real and two conjugate imaginary vertices. These are respectively the hyperbolic and elliptic cases and must be treated separately. In the hyperbolic case $k$ and $k^{\prime}$ are both real quantities, while in the elliptic case they are both complex quantities.

Let $T\left(k, k^{\prime}\right)$ and $T\left(k_{1}, k_{1}^{\prime}\right)$ be two hyberbolic transformations having the same invariant triangle. It may readily be verified from equation (3) that their resultant is $T\left(k_{2}, k_{2}{ }^{\prime}\right)$ where $k_{2}=k k_{1}$ and $k_{2}{ }^{\prime}=k^{\prime} k_{1}{ }^{\prime}$. All transformations having the same invariant triangle form a group of two parameters, $k$ and $k^{\prime}$ being the independent parameters of the group. $k$ and $k^{\prime}$ may assume in turn all possible real values; thus our group $h G_{2}(A B C)$ is continuous. 
The two parameter group $h G_{2}$ contains an infinite number of one parameter subgroups, and we proceed to determine these. All transformations in $h G_{2}$ for which the two parameters satisfy a relation of the form $k^{\prime}=k^{1-r}$, where $r$ is a constant, form a one parameter subgroup ; and conversely, in all one parameter subgroups, $k$ and $k^{\prime}$ satisfy a relation of this form. There are different subgroups for different values of $r$. Geometrically, $* r$ is interpreted as the constant cross ratio of certain four points on the tangent to a path curve of $h G_{1}(r)$, viz., the point of tangency $T$ and the points of intersection of the tangent with the sides of the invariant triangle. These four points are all real for a real hyperbolic group and hence $r$ is also real.

In order to study the distribution of the $\infty^{2}$ transformations of $h G_{2}$ into one parameter subgroups we resort to a geometrical device as follows : Let $k$ and $k^{\prime}$ be the rectangular coördinates of a point in a plane (not to be confused with the plane of our transformation). It is evident, since $k$ and $k^{\prime}$ are independent parameters, that there is a point in the plane corresponding to every transformation of the group $h G_{2}(A B C)$. Since all transformations, whose parameters $k$ and $k^{\prime}$ satisfy the relation $k^{\prime}=k^{1-r}$, form a one parameter subgroup of $h G_{2}$, we see that the curve $y=x^{1-r}$ represents this subgroup and the individual points of the curve represent the individual transformations of the group. If we give to $r$ all real values, we have a family of curves which represent the system of subgroups of $h G_{2}(A B C)$.

From the properties of this system of curves we deduce the following results: If $r$ is an irrational number, the curve $y=x^{1-r}$ contains no real point for which either coördinate is negative ; the curve lies entirely in the first quadrant. If $r$ is a rational fraction with odd numerator and odd denominator, $y$ cannot be negative, and the curve lies above the axis of $x$ in the first and second quadrants. If $r$ is rational with odd numerator and even denominator, the curve lies in the first and fourth quadrants. If $r$ is rational with even numerator and odd denominator, the curve lies in the first and third quadrants.

Every curve passes through the point $(1,1)$, which shows that the identical transformation belongs to every subgroup. The curves of our family contain every point in the first quadrant, but not every point in the second, third, and fourth quadrants. Consequently our two parameter group $h G_{2}(A B C)$ contains transformations which do not belong to any of its subgroups. Such a transformation has one or both

\footnotetext{
* Lie, "Contin. Gruppen," p. 78.
} 
of its cross ratio constants negative, and their values are such that they do not satisfy an algebraic equation of the form $k^{\prime m}=k^{n}$, where $m$ and $n$ are integers.

The variable parameter of every one parameter group in $h G_{2}(A B C)$ is $k$; and every one parameter group contains two real infinitesimal transformations, viz., when $k=1 \pm \delta$. Each infinitesimal transformation generates its corresponding portion of the group. Every transformation in the group $h G_{2}(A B C)$ for which both $k$ and $k^{\prime}$ are positive can be generated from an infinitesimal transformation of the group, while no transformation for which either $k$ or $k^{\prime}$ is negative can be so generated. Thus the group $h G_{2}$ contains three singular transformations for every non-singular $*$ one.

The failure of Lie's theory to express fully the relations here set forth for the real group $h G_{2}(A B C)$ is due to the fact that he assumes $k$ and $k^{\prime}$ to be of the form $e^{t}$ and $e^{a t}$, thus silently excluding from consideration negative values of $k$ and $k^{\prime}$.

We turn now, to the consideration of the real elliptic group $e G_{2}(A B C)$ in which the invariant triangle has one real vertex $A$ and two conjugate imaginary vertices $B$ and $C$. The invariant line $B C$ is a real line, and the one dimensional transformation along that line is elliptic, for the two invariant points on it are conjugate imaginary. The quantities $k$ and $k^{\prime}$ in equation (3) are both complex numbers; but the necessary and sufficient condition that the transformation $T$ shall be real is that $k$ and $k^{\prime}$ shall be conjugate imaginary numbers. Thus the real elliptic group $e G_{2}(A B C)$, instead of having two independent variable parameters $k$ and $k^{\prime}$, has only one, viz, $k$; but this is a complex number and may assume in turn all possible complex values. Consequently the group $e G_{2}(A B C)$ contains a transformation corresponding to every point in the complex plane.

The question of the distribution of the transformations of $e G_{2}(A B C)$ into one parameter subgroups and their generation from infinitesimal transformations of the group need not detain us long; for this group is holoedrically isomorphic with the group $G_{2}(\mathrm{mn})$ of circular transformations of the complex plane. This latter group was fully discussed by the writer in the Bulletin, Vol. 4, pp. 112-115. We learn, therefore, that every transformation of $e G_{2}(A B C)$ belongs to at least two distinct subgroups and may be gen-

* The inappropriateness of the term singular transformation is hereby shown. The term would be more appropriate if applied to those transformations in $h G_{2}(A B C)$ which do not belong to one parameter subgroups. 
erated from at least two infinitesimal transformations of the group. We sum up the discussion of type I. as follows:

THEOREM 2. Every real elliptic projective transformation of the plane belongs to some one parameter group and may be generated from the infinitesimal transformation of the group; the same is true for every hyperbolic transformation for which $k$ and $k^{\prime}$ are both positive. All other hyperbolic transformations are singular; and many of these singular transformations do not belong to one parameter groups.

Type II. - The implicit normal form of a transformation of type II. is as follows :

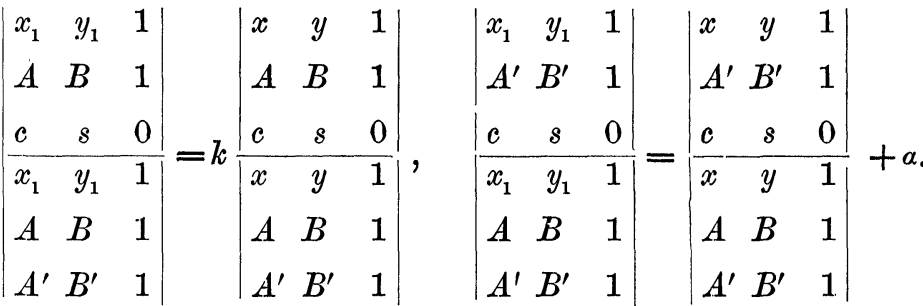

$$
\begin{aligned}
& \left(c^{2}+s^{2}=1 .\right)
\end{aligned}
$$

The invariant figure consists of two lines, their point of intersection, and a second point on one of these lines. We designate a transformation of this type by $T^{\prime}$, and its invariant figure by $(A B l)$. Along the invariant line $A B$ there is a one dimensional hyperbolic transformation whose cross ratio is $k$; while along the invariant line $A l$ is a one dimensional parabolic transformation whose constant is $\alpha$. For a real transformation $k$ and $\alpha$ are both real quantities. Two transformations $T^{\prime}(k, \alpha)$ and $T_{1}^{\prime}\left(k_{1}, \alpha_{1}\right)$ having the same invariant figure combine to form $T_{2}^{\prime}\left(k_{2}, a_{2}\right)$ where $k_{2}=$ $k k_{1}$ and $\alpha_{2}=\alpha+\alpha_{1} ; T_{2}^{\prime}$ has the same invariant figure as $T^{\prime}$ and $T_{1}^{\prime}$. Thus all transformations of type II. leaving $(A B l)$ invariant form a two parameter group $G_{2}{ }^{\prime}(A B l)$, the two parameters being $k$ and $\alpha$.

The group $G_{2}^{\prime}(A B l)$ contains $\infty^{1}$ one parameter subgroups; to show these subgroups we assume a relation between these quantities $k$ and $\alpha$, and consider only those transformations of the group $G_{2}{ }^{\prime}$ for which $k$ and $\alpha$ satisfy the assumed relation. Let $k=\alpha^{\alpha}$, where $a$ is a constant ; all transformations in $G_{2}^{\prime}$ satisfying this relation form a one parameter subgroup of $G_{2}^{\prime}, \alpha$ being the parameter. 
Within the group $G_{2}{ }^{\prime}$ there are $\infty^{1}$ such one parameter subgroups, one for each positive value of $a$.

In order to study the distribution of the transformations in $G_{2}^{\prime}$ into subgroups and their generation from infinitesimal transformations, we resort to the same device as in type I. and make $k$ and $\alpha$ the rectangular coördinates of a point in a plane. The family of curves $y=\alpha^{x}$ represents the system of one parameter subgroups of $G_{2}{ }^{\prime}$. For positive values of $a$ these curves lie in the first and second quadrants and completely fill the upper half of the plane. There are no continuous curves for negative values of $a$, and hence continuous subgroups of $G_{2}^{\prime}$ exist only for positive values of $a$.

Two particular curves of the family deserve special attention. For a very large value of $a$, the curve $y=a^{x}$ differs but little from the axis $x=0$; hence in the limit when $a=\infty$ the line $x=0$ is a curve of the family; on the other hand when $a=1$ the curve reduces to the line $y=1$. In the first case $x=0$ is the only curve of the family that penetrates into the lower half of the plane, and consequently the corresponding group is the only continuous subgroup of $G_{2}^{\prime}$ containing transformations with negative values of $k$. The transformations of the group corresponding to $a=\infty$ are in reality not of type II. but of type IV.; they are perspective transformations, the line $A l$ being the axis and the point $B$ the vertex of the transformations of the group. The transformations of the group corresponding to $a=1$ are of type V.; they are elations, the line $A B$ being the axis and $A$ the vertex of the transformations of the group.

Each one parameter subgroup of $G_{2}^{\prime}$ contains two infinitesmal transformations, one positive and the other negative. Every transformation in a subgroup of $G_{2}^{\prime}$ may be generated from one of its infinitesimal transformations, except the transformations with negative $k$ in the perspective subgroup $a=\infty$. The transformations properly of type II. in $G_{2}^{\prime}$ for which $k$ is negative do not belong to continuous subgroups of $\mathrm{G}_{2}^{\prime}$ and cannot be generated from infinitesimal transformations of the group.

TheOREM 3. All real projective transformations of type II. for which $k$ is negative are singular transformations and do not belong to one parameter groups.

Types III., IV., V.-The remaining types of projective transformations in the plane viz., types III., IV., V. may be passed over rapidly. The invariant figure of a transformation of type III. is a lineal element $A l$. There are $\infty^{3}$ 
transformations of type III. leaving invariant the same lineal element and these form a three parameter group $G_{3}{ }^{\prime \prime}$ [Lie, (13)].* This three parameter group breaks up into $\infty^{1}$ two parameter subgroups $G_{2}^{\prime \prime}[\mathrm{Lie},(24)]$, and into $\infty^{2}$ one parameter subgroups $G_{1}^{\prime \prime}[\mathrm{Lie},(37)]$. Every transformation in $G_{3}{ }^{\prime \prime}$ belongs to a one parameter subgroup and may be generated from a real infinitesimal transformation of the group. Hence there are no singular transformations of this type. I give the facts only without the proof.

Every real projective transformation of type IV. leaves invariant a real point and a real line, the vertex and axis respectively of the transformation. Every line through the vertex is an invariant line and has on it two real invariant points. Along each invariant line is a one dimensional hyperbolic transformation, and all are characterized by the same cross ratio $k$. The latter may assume in turn all real values and we thus have a one parameter group of perspective transformations. The structure of this group is identical with that of the hyperbolic group in one dimension. Hence all transformations of this group for which $k$ is negative are singular perspective transformations.

Along each of the invariant lines of a projective transformation of type $\mathrm{V}$. is a one dimensional parabolic transformation with a real constant $\alpha$. $\alpha$ may assume in turn all real values and thus we have a one parameter group of type $\mathrm{V}$. which is identical in structure with a one dimensional parabolic group. Hence there are no singular tranformations of type $\mathrm{V}$.

TheOREm 4. All real projective transformations of type IV. for which $k$ is negative are singular transformations.

It is unnecessary to discuss in detail all groups of real projective transformations in the plane. The groups containing singular transformations discussed above enter as subgroups into many of the groups of higher orders; and consequently these latter groups also contain such singular transformations. Any one who is acquainted with the structure of the projective groups of the plane can readily make the application for himself.

Kansas State University, April, 1900 .

* These numbers refer to Lie's list of perspective groups given on pages 288-291 of "Continuierliche Gruppen." 